Pacific Journal of Mathematics

FINITELY PRESENTED DIMENSION OF COMMUTATIVE 


\title{
FINITELY PRESENTED DIMENSION OF COMMUTATIVE RINGS AND MODULES
}

\author{
HO KUEN NG
}

\begin{abstract}
We define a dimension, called the finitely presented dimension, for modules and commutative rings. This dimension has nice properties when the ring in question is coherent. We then compare the finitely presented dimension with the global dimension and the weak global dimension.
\end{abstract}

Introduction. Similar to the projective and flat dimensions, we define a dimension, called the finitely presented dimension, for modules and commutative rings. It measures how far away a module is from being finitely presented, and how far away a ring is from being Noetherian.

In $\S 1$ we give the definitions and show some of the general properties. In $\S 2$, with the additional assumption of coherence, we show that the finitely presented dimension has the properties that we expect of a 'dimension'. We also point out the difference from the usual dimensions. In $\S 3$ we make a comparison of the global dimension, the weak global dimension, and the finitely presented dimension of a coherent ring; and get the relation

$$
\text { gl.dim } R=\sup (\text { w.g.dim } R, \text { f.p.dim } R-1) .
$$

We divide coherent rings into four classes according to these dimensions and show that there are 'many' rings in each class.

Throughout this paper $R$ always denotes a ring. All rings are commutative, with $1 \neq 0$. We use p.d., gl.dim, w.gl.dim to denote the projective dimension, global dimension, weak global dimension respectively. We denote the $n$th kernel of a projective resolution by $K_{n}$.

\section{Definitions and general results.}

Definition 1.1. Let $R$ be a ring and $A$ an $R$-module. We define the finitely presented dimension of $A$ (denoted by f.p. $\operatorname{dim}_{R} A$, or simply f.p.dim $A$ if the ring is clear from context) as $\inf \{n \mid$ there exists an exact sequence $P_{n+1} \rightarrow P_{n} \rightarrow \cdots \rightarrow P_{0} \rightarrow A \rightarrow 0$ of $R$-modules, where each $P_{i}$ is projective, and $P_{n+1}, P_{n}$ are finitely generated $\}$. An exact sequence giving the above infimum is called a representing sequence of $A$. If no such 
sequence exists for any $n$, we say that f.p.dim $A=\infty$. We also define the finitely presented dimension of $R$ (denoted by f.p.dim $R$ ) as $\sup \{$ f.p.dim $A \mid A$ is a finitely generated $R$-module $\}$.

Let us observe that an $R$-module $A$ is finitely presented if and only if there exists an exact sequence $F_{1} \rightarrow F_{0} \rightarrow A \rightarrow 0$, where $F_{1}, F_{0}$ are finitely generated free, if and only if there exists an exact sequence $P_{1} \rightarrow P_{0} \rightarrow A$ $\rightarrow 0$, where $P_{1}, P_{0}$ are finitely generated projective. Hence $A$ is finitely presented if and only if f.p.dim $A=0$. Consequently, we may regard our finitely presented dimension as a measure of how far away an $R$-module is from being finitely presented.

Proposition 1.2. A projective module has finitely presented dimension zero if it is finitely generated, and one otherwise.

THEOREM 1.3. $R$ is Noetherian if and only if f.p.dim $R=0$.

Proof. It follows from the fact that $R$ is Noetherian if and only if all finitely generated $R$-modules are finitely presented.

Because of this theorem, we may regard the finitely presented dimension of a ring as a measure of how far away it is from being Noetherian.

We can give bounds for the finitely presented dimensions of rings and modules.

Proposition 1.4. Let $A$ be an $R$-module. If p.d. $A \leq n$, then f.p.dim $A$ $\leq n+1$. If $\operatorname{gl} . \operatorname{dim} R \leq n$, then f.p.dim $R \leq n+1$.

Proof. It suffices to consider $n$ finite and to look at a projective resolution of $A$. The second result follows by taking the supremum of the projective dimensions of $R$-modules.

We remark that f.p.dim $R$ can be much smaller than gl.dim $R$. Take $R=Z_{4}$. The ideal (2) has projective dimension $\infty$, and hence gl.dim $R=$ $\infty$. However, $Z_{4}$ is Noetherian, so f.p.dim $R=0$.

Proposition 1.5. No finitely generated module can have finitely presented dimension 1.

Proof. Otherwise, let $A$ be such. Let $P_{2} \rightarrow P_{1} \rightarrow P_{0} \rightarrow A \rightarrow 0$ be a representing sequence of $A$, and let $K=\operatorname{Ker}\left(P_{0} \rightarrow A\right)$. Since $P_{1} \rightarrow K$ is 
surjective, $P_{1}$ is finitely generated, we have $K$ finitely generated. Now, $K$ and $A$ are both finitely generated, and so $P_{0}$ is finitely generated, because of the exact sequence $0 \rightarrow K \rightarrow P_{0} \rightarrow A \rightarrow 0$. This contradicts the fact that f.p.dim $A=1$.

\section{COROLlaRY 1.6. No ring can have finitely presented dimension 1.}

From this we see that the finitely presented dimension of a ring must be 0 or $\geq 2$. If we have a semi-simple ring, i.e. a ring with global dimension 0 , then its finitely presented dimension $\leq 1$, by 1.4 . From 1.6 we see that it must be 0 . Thus a semi-simple ring is Noetherian, a well-known result. If $R$ is a hereditary ring, i.e. a ring with global dimension 1, then its finitely presented dimension $\leq 2$. If $R$ is Noetherian, then of course f.p.dim $R=0$. If $R$ is non-Noetherian and hereditary, 1.6 shows that f.p.dim $R=2$.

Proposition 1.7. Let $A$ be an $R$-module and $S$ a multiplicatively closed set of $R$. Then

$$
\text { f.p. } \operatorname{dim}_{R} A \geq \mathrm{f} . \mathrm{p} \cdot \operatorname{dim}_{R_{S}} A_{S} \text {, }
$$

and

$$
\text { f.p.dim } R \geq \text { f.p.dim } R_{S} \text {. }
$$

Proof. If f.p. $\operatorname{dim}_{R} A=\infty$, then the first inequality follows. If it is finite, then we localize a representing sequence of $A$ at $S$, and this proves the first result. The second inequality follows from the first one, and the fact that every finitely generated $R_{S}$-module can be expressed as $A_{S}$, where $A$ is a finitely generated $R$-module.

This proposition gives the well-known result that any localization of a Noetherian ring is again Noetherian.

We note that, in general, for a finitely generated $R$-module $A$,

$$
\text { f.p.dim } A \neq \sup \left\{\text { f.p. } \operatorname{dim}_{R_{p}} A_{p} \mid p \text { is a prime in } R\right\},
$$

and

$$
\text { f.p. } \operatorname{dim} R \neq \sup \left\{\text { f.p.dim } R_{p} \mid p \text { is a prime in } R\right\} \text {. }
$$

To see this, take $R$ to be a non-Noetherian ring whose localization with respect to any prime ideal is Noetherian, e.g. the direct product of a countably infinite number of copies of $Z_{2}$. We have f.p.dim $R>0$, while 
f.p.dim $R_{p}=0$, for all prime ideals $p$ of $R$. Also, there exists a finitely generated $R$-module $A$ with f.p. $\operatorname{dim}_{R} A>0$, but f.p.dim $R_{p} A_{p}=0$, for all prime ideals $p$ of $R$.

Proposition 1.8. Let $A$ be an $R$-module, and $S$ a ring which is also a flat $R$-module. Then

$$
\text { f.p.dim }{ }_{R} A \geq \text { f.p.dim } \operatorname{dim}_{S} A \otimes_{R} S \text {. }
$$

Proof. We need only consider the case when f.p.dim ${ }_{R} A$ is finite, and tensor a representing sequence of $A$ by $S$.

Consequently, if f.p.dim $R=n$, then any finitely generated $R[X]-$ module extendable from $R$ has finitely presented dimension $\leq n$.

Proposition 1.9. Let $0 \rightarrow K \rightarrow P \rightarrow A \rightarrow 0$ be an exact sequence of $R$-modules with $P$ projective. Then

$$
\text { f.p.dim } A \leq \text { f.p.dim } K+1 \text {. }
$$

Proof. We only have to look at the case when f.p.dim $K$ is finite and combine the given exact sequence with a representing sequence of $K$.

We recall the mapping cone construction. If $F: C^{\prime} \rightarrow C$ is a morphism of complexes, then $M C(F)$ is a complex with $M C(F)_{n}=C_{n} \oplus C_{n-1}^{\prime}$. Furthermore, the sequence of complexes $0 \rightarrow C \rightarrow M C(F) \rightarrow C^{\prime}(-1) \rightarrow 0$ is exact.

We have the following facts.

If

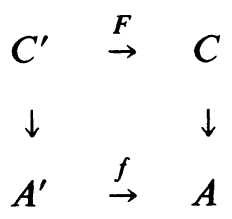

is a commutative diagram in which the vertical maps are projective resolutions, then if $f$ is a monomorphism, $M C(F)$ is a projective resolution of Coker $f$, and if $f$ is an epimorphism, then

$$
\begin{aligned}
\cdots \rightarrow & M C(F)_{n} \rightarrow M C(F)_{n-1} \rightarrow \cdots \rightarrow M C(F)_{2} \\
& \rightarrow Z_{1}(M C(F)) \rightarrow \operatorname{Ker} f \rightarrow 0
\end{aligned}
$$

is a projective resolution of $\operatorname{Ker} f$. 
TheOREM 1.10. Let $0 \rightarrow A^{\prime} \rightarrow A \stackrel{f}{\rightarrow} P \rightarrow 0$ be an exact sequence with $P$ projective.

(1) If $P$ is finitely generated, then $A^{\prime}$ is finitely presented if and only if $A$ is finitely presented.

(2) If $n \geq 1$ and f.p.dim $A^{\prime}=n$, then $1 \leq$ f.p.dim $A \leq n$.

(3) If $n \geq 1, P$ is finitely generated, and f.p.dim $A=n$, then $1 \leq$ f.p.dim $A^{\prime} \leq n$.

Thus, if $P$ is finitely generated, f.p.dim $A=$ f.p.dim $A^{\prime}$.

Proof. (1) For necessity we have $A \cong A^{\prime} \oplus P$. If $0 \rightarrow K \rightarrow F \rightarrow A^{\prime} \rightarrow 0$ is a finite presentation of $A^{\prime}$, then $0 \rightarrow K \rightarrow F \oplus P \rightarrow A^{\prime} \oplus P \rightarrow 0$ is a finite presentation of $A^{\prime} \oplus P$. For sufficiency, let $Q \rightarrow A$ be a projective resolution of $A$ with $Q_{0}, Q_{1}$ finitely generated. Note that $\cdots \rightarrow 0 \rightarrow 0 \rightarrow$ $P \rightarrow P \rightarrow 0$ is a projective resolution of $P$. Let $F$ be a map from the first resolution to the second that covers $f$. The sequence $M C(F)_{2} \rightarrow$ $Z_{1}(M C(F)) \rightarrow \operatorname{Ker} f \rightarrow 0$ is exact. $M C(F)_{2}, M C(F)_{1}$ and $M C(F)_{0}$ are finitely generated projective. The exact sequence $0 \rightarrow Z_{1}(M C(F)) \rightarrow$ $M C(F)_{1} \rightarrow M C(F)_{0} \rightarrow 0$ shows that $Z_{1}(M C(F))$ is finitely generated projective. Hence $\operatorname{Ker} f$ is finitely presented, and consequently so is $A^{\prime} \cong \operatorname{Ker} f$.

(2) follows by looking at a representing sequence of $A^{\prime}$ and direct summing $P$ to the first two terms of the sequence. That f.p.dim $A \neq 0$ follows from (1).

(3) Let $Q \rightarrow A$ be a projective resolution of $A$ with $Q_{n+1}, Q_{n}$ finitely generated. Note that $\cdots \rightarrow 0 \rightarrow 0 \rightarrow P \rightarrow P \rightarrow 0$ is a projective resolution of $P$. Let $F$ be a map from the first resolution to the second covering $f$. $M C(F)_{n+1}, M C(F)_{n+2}$ are finitely generated projective. The exact sequence $M C(F)_{n+2} \rightarrow M C(F)_{n+1} \rightarrow \cdots \rightarrow M C(F)_{2} \rightarrow Z_{1}(M C(F)) \rightarrow$ $\operatorname{Ker} f \rightarrow 0$ shows f.p.dim $\operatorname{Ker} f \leq n$, so f.p.dim $A^{\prime} \leq n$. If f.p.dim $A^{\prime}=0$, then since $P$ is finitely generated, (1) shows that f.p.dim $A=0$, which contradicts the hypothesis. Thus $1 \leq$ f.p.dim $A^{\prime} \leq n$.

The last result follows easily.

We remark that in (1) and (3), the hypothesis that $P$ be finitely generated cannot be waived, as the exact sequence $0 \rightarrow 0 \rightarrow P \rightarrow P \rightarrow 0$, where $P$ is non-finitely generated projective, shows.

Proposition 1.11. Every finitely generated $R$-module is finitely presented if and only if every cyclic $R$-module is finitely presented. 
Proof. Necessity is trivial. Sufficiency follows by picking an arbitrary ideal $I$ of $R$, looking at the exact sequence $0 \rightarrow I \rightarrow R \rightarrow R / I \rightarrow 0$, and noting that $R / I$ is a cyclic $R$-module.

In the language of finitely presented dimension, the above proposition says that $\sup \{$ f.p.dim $A \mid A$ is a cyclic $R$-module $\}=0$ if and only if f.p.dim $R=0$. This result will be generalized in $\$ 2$ under the additional hypothesis that $R$ be coherent.

2. Finitely presented dimensions of coherent rings and their modules. In this section we will justify the name 'dimension' that we have been using. If the ring in question is coherent, we show that the finitely presented dimension satisfies many theorems involving other familiar dimensions of rings. We begin by recalling some definitions.

Definition 2.1. A ring $R$ is coherent if all its finitely generated ideals are finitely presented. An $R$-module is pseudo-coherent if all its finitely generated submodules are finitely presented. An $R$-module is coherent if it is pseudo-coherent and finitely generated. [2]

PROPOSITION 2.2. The following statements are equivalent.

(1) $R$ is coherent.

(2) Every free $R$-module is pseudo-coherent.

(3) Every projective $R$-module is pseudo-coherent.

(4) For any $R$-module $A$, and for any projective resolution $P_{n+1} \rightarrow P_{n} \rightarrow$ $\cdots \rightarrow P_{0} \rightarrow A \rightarrow 0$ of $A$, with $P_{n+1}, P_{n}$ finitely generated, we can find finitely generated projective $R$-modules $P_{n+2}, P_{n+3}, \ldots$, such that $\cdots \rightarrow P_{n+3} \rightarrow$ $P_{n+2} \rightarrow P_{n+1} \rightarrow P_{n} \rightarrow \cdots \rightarrow P_{0} \rightarrow A \rightarrow 0$ is exact.

Proof. The proof of $(1) \Leftrightarrow(2)$ is contained in [3]. For $(1) \Rightarrow(3)$, see [5]. (3) $\Rightarrow(2)$ is trivial. The proofs of $(3) \Rightarrow(4)$ and $(4) \Rightarrow(2)$ are not difficult and will be omitted.

COROLlary 2.3. Let $R$ be a coherent ring. If $A$ is a finitely presented $R$-module, $P$ a finitely generated projective $R$-module, and $0 \rightarrow K \rightarrow P \rightarrow A$ $\rightarrow 0$ an exact sequence, then $K$ is finitely presented.

We make two remarks here. First, if $R$ is coherent and $A$ is an $R$-module with f.p.dim $A=n$, we have an exact sequence $P_{n+1} \rightarrow P_{n} \rightarrow$ $\cdots \rightarrow P_{0} \rightarrow A \rightarrow 0$, with each $P_{i}$ projective, and $P_{n+1}, P_{n}$ finitely generated. Then we can find $P_{n+2}, P_{n+3}, \ldots$, all finitely generated projective, such 
that $\cdots \rightarrow P_{n+3} \rightarrow P_{n+2} \rightarrow P_{n+1} \rightarrow P_{n} \rightarrow \cdots \rightarrow P_{0} \rightarrow A \rightarrow 0$ is exact. We call such an infinite exact sequence a representing sequence of $A$.

Second, if $R$ is a coherent ring, $A$ a non-finitely presented $R$-module, we look at all possible projective resolutions of $A$. If $n$ is the least integer such that $K_{n}$ is finitely generated, then f.p.dim $A=n$.

THEOREM 2.4. Let $R$ be a coherent ring, and $0 \rightarrow A^{\prime} \stackrel{f}{\rightarrow} A \stackrel{g}{\rightarrow} A^{\prime \prime} \rightarrow 0$ an exact sequence of $R$-modules. Let f.p.dim $A^{\prime}=d^{\prime}, \quad$ f.p.dim $A=d$, f.p.dim $A^{\prime \prime}=d^{\prime \prime}$. If two of these are finite, then so is the third. Furthermore, $d \leq \sup \left(d^{\prime}, d^{\prime \prime}\right), d^{\prime \prime} \leq \sup \left(d, d^{\prime}+1\right)$, and $d^{\prime} \leq \sup \left(d, d^{\prime \prime}-1\right)$.

Proof. First suppose $d^{\prime}$ and $d^{\prime \prime}$ are finite. Let $P^{\prime}, P^{\prime \prime}$ be representing sequences of $A^{\prime}, A^{\prime \prime}$, respectively. There exists a projective resolution $P$ of $A$ such that $0 \rightarrow P^{\prime} \rightarrow P \rightarrow P^{\prime \prime} \rightarrow 0$ is an exact sequence of complexes. Hence $P_{n}$ is finitely generated for $n \geq \sup \left(d^{\prime}, d^{\prime \prime}\right)$. Thus f.p.dim $A \leq$ $\sup \left(d^{\prime}, d^{\prime \prime}\right)$.

Now suppose $d^{\prime}$ and $d$ are finite. Let $P^{\prime}, P$ be representing sequences of $A^{\prime}, A$, respectively, and let $F: P^{\prime} \rightarrow P$ cover $f: A^{\prime} \rightarrow A$. Then $M C(F)$ is a projective resolution of $A^{\prime \prime}$. By the definition of $M C(F)$, we have $M C(F)_{n}$ finitely generated for all $n \geq d$ and $d^{\prime}+1$. Thus f.p.dim $A^{\prime \prime} \leq$ $\sup \left(d, d^{\prime}+1\right)$.

Finally, suppose $d$ and $d^{\prime \prime}$ are finite. Let $P, P^{\prime \prime}$ be representing sequences of $A, A^{\prime \prime}$, respectively, and let $G: P \rightarrow P^{\prime \prime}$ cover $g: A \rightarrow A^{\prime \prime}$. Then $P^{\prime}$ is a projective resolution of $A^{\prime}$, where $P_{n}^{\prime}=M C(G)_{n+1}$, for all $n \geq 1$, and $P_{0}^{\prime}=Z_{1}(M C(G)) . M C(G)_{n}$ is finitely generated for all $n \geq d^{\prime \prime}$ and $d+1$. Thus $P_{n}^{\prime}$ is finitely generated for all $n \geq d^{\prime \prime}-1$ and $d$. Also we note that $P_{0}^{\prime}$ is finitely generated, if $d^{\prime \prime} \leq 1$ and $d=0$, by the split exact sequence $0 \rightarrow Z_{1}(M C(G)) \rightarrow M C(G)_{1} \rightarrow M C(G)_{0} \rightarrow 0$. Thus f.p.dim $A^{\prime \prime}$ $\leq \sup \left(d, d^{\prime \prime}-1\right)$.

From 2.4 we get the following familiar relations.

COROLlary 2.5. With the same hypotheses as in 2.4 , we have $d \leq$ $\sup \left(d^{\prime}, d^{\prime \prime}\right)$, with equality except possibly when $d^{\prime \prime}=d^{\prime}+1$.

The following two theorems follow as consequences.

Theorem 2.6. Let $R$ be a coherent ring, and $0 \rightarrow K \rightarrow P \rightarrow A \rightarrow 0$ an exact sequence with $P$ projective. If f.p.dim $A \geq 2$, then f.p.dim $K=$ f.p.dim $A-1$. If f.p.dim $A \leq 1$, then f.p.dim $K \leq 1$. 
Theorem 2.7. Let $R$ be a coherent ring, and $0 \rightarrow K \rightarrow P \rightarrow A \rightarrow 0$ an exact sequence, with $P$ projective. If f.p.dim $K \geq 2$, then f.p.dim $K=$ f.p.dim $A-1$. If f.p.dim $K=1$, and $P$ is finitely generated, then f.p.dim $A$ $=2$. If f.p.dim $K=0$, then f.p.dim $A \leq 1$. If f.p.dim $K=0$, and $P$ is finitely generated, then f.p.dim $A=0$.

We make two further remarks here. First we note that even if $0 \rightarrow K \rightarrow P \rightarrow A \rightarrow 0$ is exact, $P$ is projective, f.p.dim $A \leq 1$, it is not necessarily true that f.p.dim $K=0$. To see this we add the exact sequence $0 \rightarrow F \rightarrow F \rightarrow 0 \rightarrow 0$ to the above sequence, where $F$ is an infinitely generated free $R$-module. Then we get a non-finitely generated kernel, which of course has f.p.dim $>0$.

Second, let $R$ be a coherent ring, $A$ an $R$-module of f.p.dim $n \geq 1$, and $P_{n+1} \rightarrow P_{n} \rightarrow \cdots \rightarrow P_{0} \rightarrow A \rightarrow 0$ an exact sequence with $P_{i}$ projective. As usual, let $K_{1}=\operatorname{Ker}\left(P_{0} \rightarrow A\right), K_{i+1}=\operatorname{Ker}\left(P_{i} \rightarrow P_{i-1}\right), i \geq 1$. Then by 2.6 f.p.dim $K_{i}=n-i, i=1,2, \ldots, n-1$. f.p.dim $K_{n}$ can be 0 or 1 . If it is finitely generated, then it is finitely presented by the coherence of $R$, and hence f.p.dim $K_{n}=0$. If it is non-finitely generated, then it has f.p.dim 1.

THEOREM 2.8. Let $R$ be a coherent ring. If $\sup \{$ f.p.dim $A \mid A$ is a cyclic $R$-module $\}=n \geq 1$, then f.p.dim $R=n$. Hence

$\sup \{$ f.p.dim $A \mid A$ is a cyclic $R$-module $\}=$ f.p.dim $R$.

Proof. The second conclusion follows from the first one and 1.11.

For the first result we note that if $n=\infty$, then it follows. Now suppose $n$ is finite. Let $A$ be a finitely generated $R$-module, say $A=$ $\left(a_{1}, \ldots, a_{s}\right)$. We need to show that f.p.dim $A \leq n$. We use induction on $s$, where the case $s=1$ follows by assumption. Assume the result holds for $s-1$. Let $\left(X_{1}, \ldots, X_{s}\right)$ be a free $R$-module mapping onto $A$ by $X_{t} \mapsto a_{i}$, $i=1, \ldots, s$. We look at the exact sequences

$$
\begin{aligned}
& 0 \rightarrow K \rightarrow\left(X_{1}, \ldots, X_{s}\right) \rightarrow\left(a_{1}, \ldots, a_{s}\right) \rightarrow 0, \\
& 0 \rightarrow L \rightarrow\left(X_{1}, \ldots, X_{s-1}\right) \rightarrow\left(a_{1}, \ldots, a_{s-1}\right) \rightarrow 0 .
\end{aligned}
$$

We note that $L \subset K,\left(X_{1}, \ldots, X_{s-1}\right) \subset\left(X_{1}, \ldots, X_{s}\right)$, and $\left(a_{1}, \ldots, a_{s-1}\right) \subset$ $\left(a_{1}, \ldots, a_{s}\right)$. Let $\bar{K}=K / L, F=\left(X_{1}, \ldots, X_{s}\right) /\left(X_{1}, \ldots, X_{s-1}\right)$, and $\bar{A}=$ $\left(a_{1}, \ldots, a_{s}\right) /\left(a_{1}, \ldots, a_{s-1}\right)$. We note that $F$ is a free $R$-module generated by the image of $X_{s}$. The sequence $0 \rightarrow \bar{K} \rightarrow F \rightarrow \bar{A} \rightarrow 0$ is exact. If f.p.dim $\bar{A}$ $=0$, then $\bar{K}$ is finitely generated, and hence is finitely presented, by the coherence of $R$. If $\left(a_{1}, \ldots, a_{s-1}\right)$ is finitely presented, then $L$ is finitely generated, and hence is finitely presented, again by the coherence of $R$. If 
f.p.dim $\bar{A} \geq 1$, it must be $\geq 2$ by 1.5 . Then f.p.dim $\bar{K}=$ f.p.dim $\bar{A}-1$ by 2.6. But this is $\leq n-1$ because $\bar{A}$ is a cyclic $R$-module. Similarly, if f.p. $\operatorname{dim}\left(a_{1}, \ldots, a_{s-1}\right) \geq 1$, we have f.p.dim $L \leq n-1$, using the induction assumption. Hence, in all cases, f.p.dim $\bar{K}$ and f.p.dim $L \leq n-1$. We look at the exact sequence $0 \rightarrow L \rightarrow K \rightarrow \bar{K} \rightarrow 0$. By 2.4,

$$
\text { f.p.dim } K \leq \sup (\text { f.p.dim } L, \text { f.p.dim } \bar{K}) \leq n-1 \text {. }
$$

From the exact sequence $0 \rightarrow K \rightarrow\left(X_{1}, \ldots, X_{s}\right) \rightarrow\left(a_{1}, \ldots, a_{s}\right) \rightarrow 0$, and 2.4 again, we have

$$
\text { f.p.dim }\left(a_{1}, \ldots, a_{s}\right) \leq \sup \left(\text { f.p.dim }\left(X_{1}, \ldots, X_{s}\right), \text { f.p.dim } K+1\right) \leq n,
$$

finishing the proof.

We consider the case when $R$ is a domain. If $A=(a)$ is a torsion-free $R$-module, then $R \cong A$ and so f.p.dim $A=0$. If $A=(a)$ is an $R$-module with torsion, then since $R$ is a domain, $a$ itself is a torsion element, and so every element of $A$ is a torsion element. Hence, if $R$ is a coherent domain, we have

$$
\text { f.p.dim } R=\sup \{\text { f.p.dim } A \mid A \text { is a cyclic torsion } R \text {-module }\} .
$$

COROLlaRY 2.9. Let $R$ be a coherent ring and $n \geq 2$. Then

$$
\sup \{\text { f.p.dim } I \mid I \text { is an ideal of } R\}=n-1
$$

if and only if f.p.dim $R=n$.

Any finitely generated ideal in a coherent ring is finitely presented. Hence the above result can be strengthened to f.p.dim $R=n$ if and only if $\sup \{$ f.p.dim $I \mid I$ is a non-finitely generated ideal of $R\}=n-1$, where $n \geq 2$.

The results that we have so far are what we expect from a 'dimension'. However, we must bear in mind that the usual 'Shifting Theorem' of dimensions does not hold when we get down to modules of dimension 1, and that we should be careful with inductive arguments because there are no finitely generated modules with dimension 1, and no rings of dimension 1.

Besides the above theorems, our finitely presented dimension also satisfies other expected relations of dimensions. We list some of these in the next proposition, the proof of which we omit. 
Proposition 2.10. Let $R$ be a coherent ring.

(1) If $0 \rightarrow A_{n} \rightarrow A_{n-1} \rightarrow \cdots \rightarrow A_{1} \rightarrow 0$ is an exact sequence, and $n-1$ of the modules $A_{1}, \ldots, A_{n}$ have finite finitely presented dimensions, then so does the remaining one.

(2) Let $\left\{A_{i} \mid i \in I\right\}$ be a family of $R$-modules with finite finitely presented dimensions. Then

$$
\text { f.p.dim } \bigoplus_{i \in I} A_{i} \geq \sup \left\{\text { f.p.dim } A_{\imath} \mid i \in I\right\} .
$$

(3) Let $A_{1}, \ldots, A_{n}$ be $R$-modules with finite finitely presented dimensions. Then f.p.dim $\left(A_{1} \oplus \cdots \oplus A_{n}\right)=\sup \left\{\right.$ f.p.dim $\left.A_{i} \mid i=1, \ldots, n\right\}$.

(4) Let $A$ be an R-module. Let $a_{1}, \ldots, a_{n}$ be an $R$-sequence on $A$. Then f.p.dim $\left(A /\left(a_{1}, \ldots, a_{n}\right) A\right) \leq n+$ f.p.dim $A$.

We remark that (3) fails for an infinite direct sum. To see this consider the following example, where $R$ is a coherent ring. We have f.p.dim $\operatorname{dim}_{R} R=0$. Let $F$ be an infinite direct sum of copies of $R$. Then f.p. $\operatorname{dim}_{R} F=1$.

The next theorem will be useful in $\S 3$ to produce examples.

THEOREM 2.11. Let $R, S$ be coherent rings. Then

$$
\text { f.p.dim }(R \oplus S)=\sup (\text { f.p.dim } R, \text { f.p.dim } S) .
$$

Proof. First let f.p.dim $R=m<\infty$, f.p.dim $S=n<\infty, m \geq n$. Let $A$ be a finitely generated $(R \oplus S)$-module. Then $A=B \oplus C$, where $B$ is a finitely generated $R$-module, and $C$ is a finitely generated $S$-module. We have exact sequences

$$
\begin{aligned}
& P_{m+1} \rightarrow P_{m} \rightarrow \cdots \rightarrow P_{0} \rightarrow B \rightarrow 0, \\
& Q_{m+1} \rightarrow Q_{m} \rightarrow \cdots \rightarrow Q_{0} \rightarrow C \rightarrow 0,
\end{aligned}
$$

of $R$-modules and $S$-modules respectively, where the $P_{i}$ 's are $R$-projective, $P_{m+1}, P_{m} R$-finitely generated, and the $Q_{i}$ 's are $S$-projective, $Q_{m+1}, Q_{m}$ $S$-finitely generated. Regarding these as exact sequences of $(R \oplus S)$ modules, we have an exact sequence of $(R \oplus S)$-modules

$$
P_{m+1} \oplus Q_{m+1} \rightarrow P_{m} \oplus Q_{m} \rightarrow \cdots \rightarrow P_{0} \oplus Q_{0} \rightarrow B \oplus C \rightarrow 0,
$$

where the $\left(P_{i} \oplus Q_{i}\right)$ 's are $(R \oplus S)$-projective, and $P_{m+1} \oplus Q_{m+1}, P_{m} \oplus Q_{m}$ are $(R \oplus S)$-finitely generated. So f.p.dim $\operatorname{dim}_{R \oplus S} A \leq m$. Thus

$$
\text { f.p.dim }(R \oplus S) \leq \sup (\text { f.p.dim } R, \text { f.p.dim } S) .
$$


Suppose f.p.dim $(R \oplus S)=k$ and $k<m$. Let $B$ be a finitely-generated $R$-module with f.p.dim $\operatorname{dim}_{R} B>k$. We can regard $B$ as an $(R \oplus S)$-module by defining $(r, s) b=r b$, for $r \in R, s \in S$, and $b \in B$. Then $(1,0) B \cong B$, as $R$-modules. As an $(R \oplus S)$-module, we have an exact sequence $P_{k+1} \rightarrow$ $P_{k} \rightarrow \cdots \rightarrow P_{0} \rightarrow B \rightarrow 0$, where each $P_{i}$ is $(R \oplus S)$-projective, and $P_{k+1}$, $P_{k}$ are $(R \oplus S)$-finitely generated. Thus

$$
(1,0) P_{k+1} \rightarrow(1,0) P_{k} \rightarrow \cdots \rightarrow(1,0) P_{0} \rightarrow(1,0) B \rightarrow 0
$$

is an exact sequence of $R$-modules, where each $(1,0) P_{i}$ is $R$-projective, and $(1,0) P_{k+1},(1,0) P_{k}$ are $R$-finitely generated. Then f.p. $\operatorname{dim}_{R} B \leq k$, a contradiction. Thus

$$
\text { f.p.dim }(R \oplus S)=\sup (\text { f.p.dim } R, \text { f.p.dim } S) .
$$

The second part of the above proof also shows that if one of f.p.dim $R$, f.p.dim $S$ is infinite, then so is f.p.dim $(R \oplus S)$. This completes the proof.

3. Relations with other dimensions. In this section we obtain some results relating the finitely presented, global, and weak global dimensions.

Up to now the only result we have is 1.4 , namely, f.p.dim $R \leq 1+$ gl.dim $R$.

First, a familiar fact is that for a Noetherian ring $R$, we have gl.dim $R=$ w.gl.dim $R$. We have the following generalization.

Proposition 3.1. Let $R$ be a coherent ring, with w.gl.dim $R \geq$ f.p.dim $R$. Then gl.dim $R=$ w.gl.dim $R$.

Proof. It suffices to prove that w.gl.dim $R \geq \operatorname{gl} . \operatorname{dim} R$, and we may assume w.gl.dim $R$ is finite. We need only consider

$$
2 \leq \mathrm{f} . \mathrm{p} \cdot \operatorname{dim} R \leq \mathrm{w} \cdot \mathrm{gl} \cdot \operatorname{dim} R=n<\infty,
$$

by the previous remark. For any finitely-generated $R$-module, we can find a resolution such that the $n$th kernel is finitely presented flat, and hence projective.

LEMMA 3.2. If $R$ is a coherent ring, and f.p.dim $R>$ w.gl.dim $R$, then gl.dim $R=$ f.p.dim $R$ or f.p.dim $R-1$.

Proof. If f.p.dim $R=\infty$, the result follows from 1.4. Now suppose f.p.dim $R=n<\infty$. Note that $n \geq 2$ because $n=$ f.p.dim $R>$ w.gl.dim $R$ $\geq 0$. An argument like the one used in the proof of 3.1 shows that 
gl.dim $R \leq n=$ f.p.dim $R$. By 1.4 again, f.p.dim $R \leq 1+\operatorname{gl} \cdot \operatorname{dim} R$. Hence,

$$
\text { gl.dim } R=\text { f.p.dim } R \text { or f.p.dim } R-1 \text {. }
$$

Proposition 3.3. Let $R$ be a coherent ring with gl.dim $R>$ w.gl.dim $R$. Then f.p.dim $R=1+$ gl.dim $R$.

Proof. First it follows from 3.1 and 3.2 that f.p.dim $R=1+$ gl.dim $R$ or gl.dim $R$. Thus we need only consider gl.dim $R=n<\infty$ and show that f.p.dim $R=$ gl.dim $R$ is impossible. Else let $A$ be a finitely generated $R$-module. We can find an exact sequence $0 \rightarrow P_{n} \rightarrow P_{n-1} \rightarrow \cdots \rightarrow P_{0} \rightarrow A$ $\rightarrow 0$, with each $P_{i}$ projective, and $P_{n}$ finitely presented. Let $K_{n-1}$ be the $(n-1)$ th kernel. Observe that $n$ cannot be 1 . Also note that $n>$ w.gl.dim $R \geq 0$. We consider the exact sequence $0 \rightarrow P_{n} \rightarrow P_{n-1} \rightarrow K_{n-1}$ $\rightarrow 0$. As w.gl.dim $R<\operatorname{gl} . \operatorname{dim} R=n, K_{n-1}$ is flat. By projectivity, let $Q$ be such that $P_{n-1} \oplus Q=F$ is free. Then $0 \rightarrow P_{n} \rightarrow F \rightarrow K_{n-1} \oplus Q \rightarrow 0$ is exact, and $K_{n-1} \oplus Q$ is flat. Let $p_{1}, \ldots, p_{m}$ generate $P_{n}$. Using the flatness of $K_{n-1} \oplus Q$, there exists a homomorphism $F \rightarrow P_{n}$ such that $p_{i} \mapsto p_{i}$, for $i=1, \ldots, m$, by [3]. Thus the above short exact sequence splits, and so $F \cong P_{n} \oplus K_{n-1} \oplus Q$. Thus $K_{n-1}$ is projective. Therefore p.d. $A \leq n-1$, so gl.dim $R \leq n-1$, a contradiction. Hence, f.p.dim $R \neq \operatorname{gl} . \operatorname{dim} R$, and so we must have f.p.dim $R=1+$ gl.dim $R$.

Consequently, if $R$ is coherent, w.gl.dim $R<\operatorname{gl} . \operatorname{dim} R=n<\infty$, we can find a finitely generated $R$-module, or even a cyclic $R$-module, such that in all projective resolutions of this module, the first $n$ kernels are non-finitely generated.

The above proposition can be stated simply and clearly as

THEOREM 3.4. For a coherent ring $R$, we have gl.dim $R=\sup ($ w.gl.dim $R$, f.p.dim $R-1)$.

The following particular case is worth noting.

Corollary 3.5. Let $R$ be a Prüfer domain.

(1) If f.p.dim $R=m \geq 2$, then $\operatorname{gl} \cdot \operatorname{dim} R=m-1$.

(2) If gl.dim $R=n \geq 2$, then f.p.dim $R=n+1$.

Proof. We know that a Prüfer domain has w.gl.dim $\leq 1$. Also, a Prüfer domain is coherent, since a non-zero finitely generated ideal is invertible, hence projective, and hence finitely presented. Now (1) and (2) follow immediately from 3.4 . 
In [4] it was shown that given any $n$, there exists a valuation ring $R$ with gl.dim $R=n$. We can show the following result easily.

Proposition 3.6. For any $n \neq 1,2, n \geq 0$, there exists a valuation ring $R$ with f.p.dim $R=n$.

Proof. If $n=0$, we take $R$ to be a discrete valuation ring. For $n>2$, let $R$ be a valuation ring of global dimension $n-1$. As $n-1 \geq 2$, f.p.dim $R=n$, by $3.5(2)$, since a valuation ring is clearly Prüfer.

We remark that there does not exist a valuation $\operatorname{ring} R$ with f.p.dim $R$ $=1$, by the more general result 1.6. Also, there does not exist a valuation ring $R$ with f.p.dim $R=2$. Otherwise, we must have $\operatorname{gl} . \operatorname{dim} R=1$, by 3.5(1). Then any non-zero ideal of $R$ is projective, hence invertible, and hence finitely generated. Thus $R$ is Noetherian, so f.p.dim $R=0$, a contradiction.

From the foregoing discussion, we see that the weak global, global, and finitely presented dimensions of a coherent ring $R$ must satisfy one of the following mutually exclusive complementary diagrams.

Case 1:

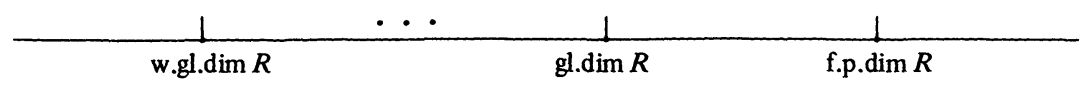

Case 2:

w.gl.dim $R=$ gl.dim $R=$ f.p.dim $R$

Case 3:

$\frac{1}{\text { f.p.dim } R} \frac{1}{\text { w.gl.dim } R=\operatorname{gl} \cdot \operatorname{dim} R}$

Case 4:

$\frac{1}{\text { w.gl.dim } R=\text { gl.dim } R} \frac{1}{\text { f.p.dim } R}$

In the diagrams,

represents two consecutive numbers, while

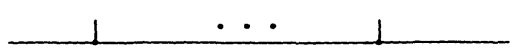

means that the numbers may not be consecutive. 
We now show that all four cases are possible.

For convenience we call a ring $R$ an $(a, b, c)$-ring, if w.gl.dim $R=a$, gl.dim $R=b$, and f.p.dim $R=c$. We observe that if $R$ is a coherent $\left(a_{1}, b_{1}, c_{1}\right)$-ring, and $S$ is a coherent $\left(a_{2}, b_{2}, c_{2}\right)$-ring, then $R \oplus S$ is a coherent $\left(\sup \left(a_{1}, a_{2}\right), \sup \left(b_{1}, b_{2}\right), \sup \left(c_{1}, c_{2}\right)\right)$-ring.

An example of Case 1 is a von Neumann regular, hereditary, nonNoetherian ring, i.e. a $(0,1,2)$-ring. Such a ring can be found in [1].

An example of Case 2 is a semi-simple ring, i.e. a (0,0,0)-ring.

An example of Case 3 is a regular local ring with global dimension $n \geq 1$, i.e. an $(n, n, 0)$-ring.

An example of Case 4 is the direct sum of the example of Case 1 and a regular local ring of global dimension 1 , since the direct sum of a $(0,1,2)$-ring and $a(1,1,0)$-ring is a $(1,1,2)$-ring.

We note that all these rings are coherent.

Now we give more examples of Cases 1-4 in 3.7-3.10, respectively. First we recall that, by 3.6, for any integer $n \geq 2$, there is a valuation ring with weak global dimension $\leq 1$, global dimension $n$, and finitely presented dimension $n+1$.

Proposition 3.7. Let $1 \leq m<n$, where $m$ and $n$ are integers. Then $a$ coherent $(m, n, n+1)$-ring exists.

Proof. We take a regular local ring of global dimension $m$, i.e. an $(m, m, 0)$-ring. The direct sum of this and a valuation ring of global dimension $n$ is a coherent $(m, n, n+1)$-ring.

Proposition 3.8. Let $n$ be an integer, $n \geq 0, n \neq 1$. Then a coherent $(n, n, n)$-ring exists.

Proof. A semi-simple ring is $(0,0,0)$. The direct sum of a coherent $(0,1,2)$-ring and a regular local ring of global dimension 2 , i.e. a $(2,2,0)$ ring, is a $(2,2,2)$-ring. For $n>2$ we have a valuation ring of global dimension $n-1$ by 3.6. The direct sum of this ring and a regular local ring of global dimension $n$, i.e. an $(n, n, 0)$-ring, gives an $(n, n, n)$-ring. It is clear that all these rings are coherent.

Proposition 3.9. Let $m, n$ be integers, $m>n \geq 0, n \neq 1$. Then $a$ coherent $(m, m, n)$-ring exists. 
Proof. We know that a regular local ring of global dimension $m$ is a coherent $(m, m, 0)$-ring. By 3.8 a coherent $(n, n, n)$-ring exists. The direct sum is a coherent $(m, m, n)$-ring.

Proposition 3.10. Let $n$ be an integer, $n \geq 1$. Then a coherent $(n, n, n+1)$-ring exists.

Proof. First we let $n>1$. By 3.6 there is a valuation ring with global dimension $n$. The direct sum of this ring with a regular local ring of global dimension $n$ gives a coherent $(n, n, n+1)$-ring. If $n=1$ then the direct sum of a $(0,1,2)$-ring and a regular local ring of global dimension 1 gives a coherent $(1,1,2)$-ring.

In all these examples, instead of a regular local ring of global dimension $n$, we may take the polynomial ring of $n$ indeterminates over a field. Both are $(n, n, 0)$-rings.

Using our previous results a theorem on polynomial extension can be established.

THEOREM 3.11. Let $R[X]$ be coherent, and w.gl.dim $R<\operatorname{gl} . \operatorname{dim} R$. Then f.p.dim $R[X]=1+$ f.p.dim $R$.

Proof. $R$ cannot be Noetherian. We observe that $R \cong R[X] /(X)$ is also coherent, and that we are in Case 1 . The result follows easily.

Acknowledgements. The author would like to thank Professor A. Seidenberg for his advice and the referee for valuable suggestions.

\section{REFERENCES}

[1] G. M. Bergman, Hereditary commutative rings and centres of hereditary rings, Proc. London Math. Soc., (3) 23 (1971), 214-236.

[2] N. Bourbaki, Algèbre Commutative, Hermann, Paris, 1961-1965.

[3] S. U. Chase, Direct product of modules, Trans. Amer. Math. Soc., 97 (1960), 457-473.

[4] B. L. Osofsky, Global dimension of valuation rings, Trans. Amer. Math. Soc., 127 (1967), 136-149.

[5] J. P. Soublin, Anneaux et modules cohérents, J. Algebra, 15 (1970), 455-472.

Received November 2, 1982. 



\section{PACIFIC JOURNAL OF MATHEMATICS \\ EDITORS}

Donald BaBBITT (Managing Editor)

University of California

Los Angeles, CA 90024

Hugo RossI

University of Utah

Salt Lake City, UT 84112

C. C. Moore and Arthur Ogus

University of California

Berkeley, CA 94720
J. DugundiI

Department of Mathematics

University of Southern California

Los Angeles, CA 90089-1113

R. FINN and H. SAMELSON

Stanford University

Stanford, CA 94305

\section{ASSOCIATE EDITORS}
R. ARENS
E. F. BECKENBACH
B. H. NeUMANN
F. WOLF
K. YoshidA (1906-1982)

\section{SUPPORTING INSTITUTIONS}

\author{
UNIVERSITY OF ARIZONA \\ UNIVERSITY OF BRITISH COLUMBIA \\ CALIFORNIA INSTITUTE OF TECHNOLOGY \\ UNIVERSITY OF CALIFORNIA \\ MONTANA STATE UNIVERSITY \\ UNIVERSITY OF NEVADA, RENO \\ NEW MEXICO STATE UNIVERSITY \\ OREGON STATE UNIVERSITY
}

\author{
UNIVERSITY OF OREGON \\ UNIVERSITY OF SOUTHERN CALIFORNIA \\ STANFORD UNIVERSITY \\ UNIVERSITY OF HAWAII \\ UNIVERSITY OF TOKYO \\ UNIVERSITY OF UTAH \\ WASHINGTON STATE UNIVERSITY \\ UNIVERSITY OF WASHINGTON
}

The Supporting Institutions listed above contribute to the cost of publication of this Journal, but they are not owners or publishers and have no responsibility for its content or policies.

Mathematical papers intended for publication in the Pacific Journal of Mathematics should be in typed form or offset-reproduced (not dittoed), double spaced with large margins. Please do not use built up fractions in the text of the manuscript. However, you may use them in the displayed equations. Underline Greek letters in red, German in green, and script in blue. The first paragraph must be capable of being used separately as a synopsis of the entire paper. In particular it should contain no bibliographic references. Please propose a heading for the odd numbered pages of less than 35 characters. Manuscripts, in triplicate, may be sent to any one of the editors. Please classify according to the scheme of Math. Reviews, Index to Vol. 39. Supply name and address of author to whom proofs should be sent. All other communications should be addressed to the managing editor, or Elaine Barth, University of California, Los Angeles, California 90024.

There are page-charges associated with articles appearing in the Pacific Journal of Mathematics. These charges are expected to be paid by the author's University, Government Agency or Company. If the author or authors do not have access to such Institutional support these charges are waived. Single authors will receive 50 free reprints; joint authors will receive a total of 100 free reprints. Additional copies may be obtained at cost in multiples of 50 .

The Pacific Journal of Mathematics is issued monthly as of January 1966. Regular subscription rate: $\$ 132.00$ a year (6 Vol., 12 issues). Special rate: $\$ 66.00$ a year to individual members of supporting institutions.

Subscriptions, orders for numbers issued in the last three calendar years, and changes of address should be sent to Pacific Journal of Mathematics, P.O. Box 969, Carmel Valley, CA 93924, U.S.A. Old back numbers obtainable from Kraus Periodicals Co., Route 100, Millwood, NY 10546.

The Pacific Journal of Mathematics ISSN 0030-8730 is published monthly by the Pacific Journal of Mathematics at P.O. Box 969, Carmel Valley, CA 93924. Application to mail at Second-class postage rates is pending at Carmel Valley, California, and additional mailing offices. Postmaster: Send address changes to Pacific Journal of Mathematics, P. O. Box 969, Carmel Valley, CA 93924.

PUBLISHED BY PACIFIC JOURNAL OF MATHEMATICS, A NON-PROFIT CORPORATION

Copyright $\odot 1984$ by Pacific Journal of Mathematics 


\section{Pacific Journal of Mathematics}

\section{Vol. 113, No. $2 \quad$ April, 1984}

Alan Adolphson, On the Dwork trace formula ...................257

Amos Altshuler and Leon Steinberg, Enumeration of the quasisimplicial

3 -spheres and 4-polytopes with eight vertices .................. 269

Kenneth R. Goodearl, Cancellation of low-rank vector bundles .......... 289

Gary Fred Gruenhage, Ernest A. Michael and Yoshio Tanaka, Spaces

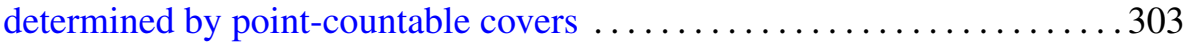

Charles Lemuel Hagopian, Atriodic homogeneous continua .......... 333

David Harbater, Ordinary and supersingular covers in characteristic $p$. . . 349

Domingo Antonio Herrero, Continuity of spectral functions and the lakes

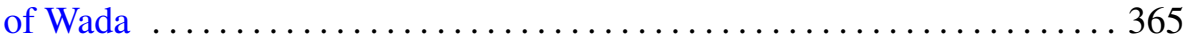

Donald William Kahn, Differentiable approximations to homotopy resolutions and framed cobordism ....................... 373

K. McGovern, On the lifting theory of finite groups of Lie type $\ldots \ldots \ldots . \ldots 383$

C. David (Carl) Minda, The modulus of a doubly connected region and the geodesic curvature-area method ............................. 395

Takuo Miwa, Complexes are spaces with a $\sigma$-almost locally finite base $\ldots . .407$

Ho Kuen Ng, Finitely presented dimension of commutative rings and modules

Roger David Nussbaum, A folk theorem in the spectral theory of

$C_{0}$-semigroups

J. S. Okon, Prime divisors, analytic spread and filtrations

Harold Raymond Parks, Regularity of solutions to elliptic isoperimetric problems

R. Sitaramachandra Rao and M. V. Subba Rao, Transformation formulae for multiple series

Daniel Ruberman, Imbedding punctured lens spaces and connected sums

Uri Srebro, Deficiencies of immersions 